\title{
The influence of the new ECMWF Ensemble Prediction System resolution on wind power forecast accuracy and uncertainty estimation
}

\author{
S. Alessandrini ${ }^{1}$, S. Sperati ${ }^{1}$, and P. Pinson ${ }^{2}$ \\ ${ }^{1}$ RSE, Ricerca Sistema Energetico, Milano, Italy \\ ${ }^{2}$ Technical University of Denmark (DTU), DTU Informatics, Lyngby, Denmark \\ Correspondence to: S. Alessandrini (stefano.alessandrini@rse-web.it)
}

Received: 17 January 2012 - Revised: 14 May 2012 - Accepted: 21 June 2012 - Published: 27 July 2012

\begin{abstract}
The importance of wind power forecasting (WPF) is nowadays commonly recognized because it represents a useful tool to reduce problems of grid integration and to facilitate energy trading. If on one side the prediction accuracy is fundamental to these scopes, on the other it has become also clear that a reliable estimation about their uncertainty is paramount. In fact prediction accuracy is unfortunately not constant and can depend on the location of a particular wind farm, on the forecast time and on the atmospheric situation. Previous studies indicated that the spread of power forecasts derived from the Ensemble Prediction System (EPS) in use at the European Centre for Medium-Range Weather Forecast (ECMWF) could be used as indicator of a three-hourly, three days ahead, wind power forecast's accuracy. In this paper a new application of the EPS, whose horizontal resolution was increased on January 2010 from T399/T255 (60 km) to T639/T319 (32 km), shows an improvement in the results implying that the power spread has actually enough correlation with the error calculated on the deterministic forecast in order to be used as an accuracy predictor. The periods for this comparison are from January 2008 until October 2008 (T399/T255) and from January 2011 until October 2011 (T639/T319). Moreover we have focused our attention on the influence of the new EPS configuration on the performance of a deterministic WPF conducted with the ensemble mean: the results show that increasing the EPS resolution yields a single-valued WPF whose performance is comparable with that of the new ECMWF deterministic high-resolution meteorological model, whose spatial resolution increased from T799 $(25 \mathrm{~km})$ to $\mathrm{T} 1279(15 \mathrm{~km})$.
\end{abstract}

\section{Introduction}

Short-term forecasting of wind energy production is becoming increasingly important due to the constant growth of this renewable source, whose uncertainty require a significant commitment to meet the needs of the transmission networks operators and of other agents of the power systems and electricity markets. Wind is in fact a variable energy source, which does not allow a management similar to that of other traditional sources: in order to be able to better control its integration into the electric grids and its dispatching priority, an adequate insight of its dynamics is necessary, in particular for the short-term time horizon $(0-48 \mathrm{~h})$ in which trading operations are carried out in the electricity markets. The accuracy of a short-term wind power forecast is consequently a raising concern (Pinson et al., 2007; Holmgren et al., 2010). On the basis of these considerations, our application investigated the performances of the new resolution ECMWF deterministic and EPS models applied to the same wind farm as in previous work (Alessandrini et al., 2011), comparing them with the results obtained with the previous lower resolution versions of these two models. The new versions have been applied to understand if it is possible to increase the performance in both wind power prediction accuracy and uncertainty estimation. 
Table 1. Mean and standard deviation of measured wind speed $\left(\mathrm{m} \mathrm{s}^{-1}\right)$ and power $(\mathrm{kW})$ for the two periods.

\begin{tabular}{llrr}
\hline & & 2008 & 2011 \\
\hline \multirow{2}{*}{ Wind speed } & Mean & 6.69 & 6.71 \\
& Standard deviation & 3.09 & 3.16 \\
\hline \multirow{2}{*}{ Power } & Mean & 210.02 & 204.37 \\
& Standard deviation & 225.21 & 222.71 \\
\hline
\end{tabular}

\section{Site and wind data description}

The wind farm used in the application is located in a complex-terrain mountain area of Northern Sicily. It has 9 equal turbines, each one of $850 \mathrm{~kW}$ Nominal Power (NP). Time series power data for the single turbines are available for the years 2008 and 2011, in each case covering the period January-October. To consider valid power data we computed an average over the active turbines for each time step obtaining a single power series. Wind data has been measured at $50 \mathrm{~m}$ a.g.l. (same height of the hubs) by an anemometer located inside the park. Measured data have been averaged from 10-minutes timesteps to hourly periods.

The total fraction of missing hourly data is similar and lower than $5 \%$ for both periods of the comparison. Some concerns may rise regarding the fact that we compared two models with different resolution on two different years instead of the same period. However, the mean and standard deviation of measured wind speed and power reported in Table 1 are quite similar. This allowed us to consider the two periods suitable enough for the comparison, using the same forecast system and post-processing configuration for both cases.

\section{The wind power forecast system}

The wind power forecast system used consists in the application of the ECMWF meteorological model, which produces three-hourly wind forecasts at $10 \mathrm{~m}$ a.g.l., for the three days ahead period and for each year using respectively the two different model resolutions. In order to reduce systematic errors and, at the same time, to convert wind to power, a singlelayer, feedforward Neural Network (NN) has been applied (Cali et al., 2008). The NN has been configured linking directly the forecasted meteorological data with the measured power on the training period, in order to obtain a three-hourly forecast power series. This WPF structure has been applied also in Alessandrini et al. (2010) showing similar or better performances to those of other WPF topology (i.e. Numerical Weather Prediction + Model Output Statistic (MOS) correction + power curve).

\section{The deterministic application}

In both cases the first 4 months have been used as training period for the NN described above, while the remaining 6 months have been considered as test period. However, the $\mathrm{NN}$ application is recursive (i.e. self-adapting algorithm that uses recent errors to correct its weights at regular intervals), therefore the training period is continuously increased including the part of the test period previous to the forecast window. The performances have been evaluated with some statistical indices reported in Table 2, in which RMSE, MAE and bias are normalized by NP. The indices are calculated averaging over all the three hours forecast horizon between time $0 \mathrm{~h}$ to $+24 \mathrm{~h}$ (Day 1 ), $+24 \mathrm{~h}$ to $+48 \mathrm{~h}$ (Day 2 ) and $+48 \mathrm{~h}$ to $+72 \mathrm{~h}$ (Day 3) where $0 \mathrm{~h}$ refers to 00:00 UTC, the beginning of each 3 days forecast run updated every $24 \mathrm{~h}$. On the first day prediction the new resolution shows a small improvement in terms of RMSE apart from that the other indices look comparable, suggesting that the increase in resolution does not necessary assure better performances. Looking at the MAE indices, the results are in both cases in line with that of other state of the art models applied on complex terrain cases (Giebel et al., 2011).

\section{The probabilistic application}

The ECMWF EPS members are generated with perturbation of the initial conditions (ECMWF report, 2010) using singular vectors with maximum total energy growth, obtaining 50 pair of wind components plus a non-perturbed member (the control run). The 50+1 wind forecasts have been first processed by a MOS (correction), training a NN on the ensemble mean and then applying it recursively on the test period. In this case the NN (with the same structure as indicated in Sect. 4) has been configured to link the wind forecasted data with measured wind speed instead of wind power, as done in the deterministic application, in order to obtain $50+1$ corrected wind speed ensemble members, on which performing a statistical calibration procedure (Pinson, 2012).

The procedure employed is based on logit-transformation of the wind speed forecasted and measured data in order to reproduce a Gaussian distribution; the ratio between the RMSE, calculated on the training period between measured wind speed and the MOS-corrected ensemble mean, and the mean of the standard deviation of the ensemble members has been used as a variance deficit to adaptively correct the mean and the variance of the transformed wind speed forecasts on the test period. The time series have been finally transformed back with the inverse logit-transformation.

The rank histograms before calibration show in both cases an overconfident model, with the measurements falling quite often outside the ensemble distribution. The situation is improved by the calibration procedure, showing a more uniform distribution between the various bins. However, the extreme bins still remain more populated than the others, as 
Table 2. Statistical indices for three-hourly power forecast evaluation computed using measured data for the two case periods. RMSE, MAE and bias are normalized by the nominal power (NP). The indices are calculated averaging over all the three hours forecast horizon between time $0 \mathrm{~h}$ to $+24 \mathrm{~h}$ (Day 1 ),$+24 \mathrm{~h}$ to $+48 \mathrm{~h}$ (Day 2) and $+48 \mathrm{~h}$ to $+72 \mathrm{~h}$ (Day 3) where $0 \mathrm{~h}$ refers to 00:00 UTC, the beginning of each 3 days forecast run updated every $24 \mathrm{~h}$.

\begin{tabular}{lllll}
\hline & & Day 1 & Day 2 & Day 3 \\
\hline Deterministic T799 $(25 \mathrm{~km})$ & RMSE/NP & $14.5 \%$ & $15.2 \%$ & $16.0 \%$ \\
& MAE/NP & $10.3 \%$ & $10.6 \%$ & $11.7 \%$ \\
& Pearson Correlation & 0.77 & 0.75 & 0.71 \\
& bias/NP & -0.02 & -0.01 & 0.0 \\
\hline Deterministic T1279 (15km) & RMSE/NP & $13.8 \%$ & $15.5 \%$ & $16.0 \%$ \\
& MAE/NP & $10.1 \%$ & $11.2 \%$ & $12.1 \%$ \\
& Pearson Correlation & 0.78 & 0.71 & 0.69 \\
& bias/NP & 0.01 & 0.01 & 0.02 \\
\hline EPS mean T399/T255 (60 km) & RMSE/NP & $15.3 \%$ & $15.8 \%$ & $16.7 \%$ \\
& MAE/NP & $11.1 \%$ & $11.7 \%$ & $12.6 \%$ \\
& Pearson Correlation & 0.74 & 0.71 & 0.67 \\
& bias/NP & -0.02 & -0.01 & 0.0 \\
\hline EPS mean T639/T319 (32 km) & RMSE/NP & $14.3 \%$ & $15.6 \%$ & $15.9 \%$ \\
& MAE/NP & $10.2 \%$ & $11.2 \%$ & $11.7 \%$ \\
& Pearson Correlation & 0.76 & 0.70 & 0.69 \\
& bias/NP & 0.0 & 0.0 & 0.0 \\
\hline
\end{tabular}

EPS T399/T255

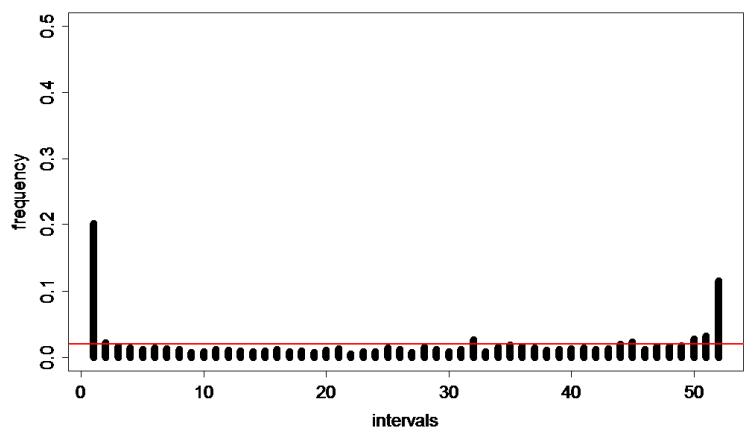

EPS T639/T319

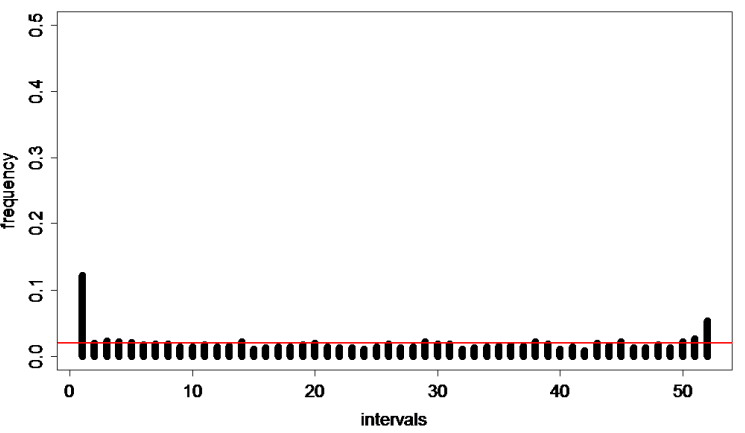

Figure 1. Rank histograms, calibrated wind speed ensemble members; EPS T399/T255 and EPS T639/T319 resolution.

Table 3. Correlation between power spread averaged on daily basis and daily RMSE, and ratio between cases falling simultaneously above or below their respective median value and total number of cases (Day 1, 2 and 3 interval are explained in Table 2 caption).

\begin{tabular}{lllllllll}
\hline & \multicolumn{2}{c}{ spread/error correlation } & & \multicolumn{3}{c}{ diagonal ratio } \\
\cline { 2 - 3 } \cline { 6 - 8 } & Day 1 & Day 2 & Day 3 & & Day 1 & Day 2 & Day 3 \\
\hline 2008 & 0.57 & 0.55 & 0.57 & & 0.66 & 0.66 & 0.66 \\
2011 & 0.5 & 0.68 & 0.63 & & 0.71 & 0.73 & 0.73 \\
\hline
\end{tabular}

shown in Fig. 1. It is noticeable that for 2011 the MOS (system) + calibration scheme seems to work slightly better than for the 2008 case. There are probably two reasons for that. First the increase in spatial resolution slightly im- proves forecast accuracy. Secondarily the initial ensemble spread of the higher resolution model is in general greater than in the low resolution model requiring a lower job from the calibration process. The calibrated ensemble members are then converted to wind power using a theoretical power curve provided by the manufacturer. In Figs. 2 and 3 we show some contingency diagrams (Von Bremen, 2007): the graphs plot the daily RMSE/NP of the deterministic WPF versus the ensemble power spread, expressed as standard deviation of the ensemble power members, for the three-days period. The 2011 application shows an increase in the performances, the diagonal cases (those when the spread and forecast errore are simultaneously above or below their respective median values) are in fact more populated than the off-diagonal cases meaning that at a low ensemble spread is more often related to low forecast errors. Table 3 shows correlation 

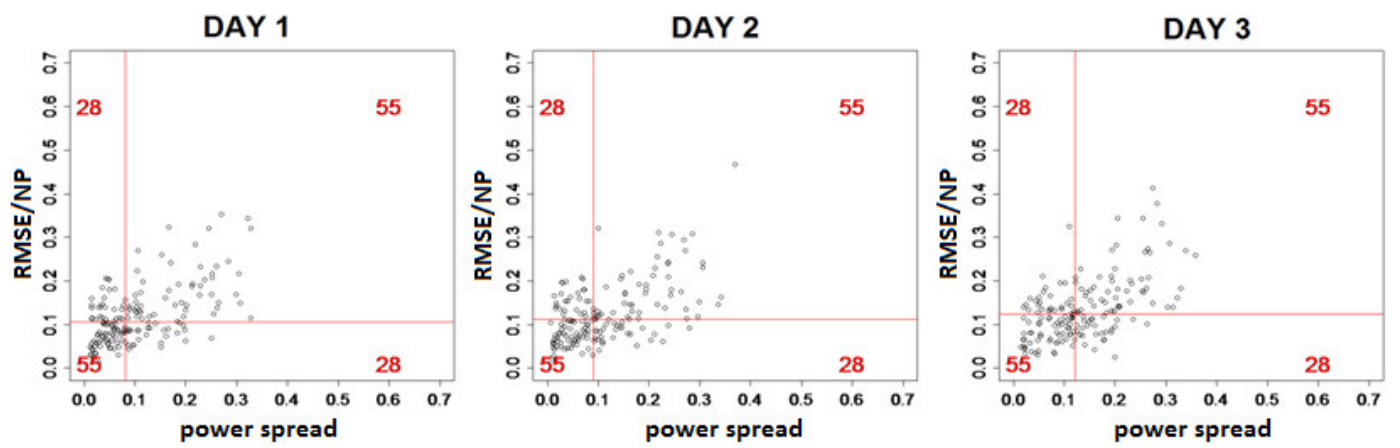

Figure 2. ECMWF deterministic power forecast error (RMSE/NP) vs. ensemble power spread computed as a daily average for three lead times. EPS T399/T255 $(60 \mathrm{~km})$. The lines are plotted at the statistical median value of the data reported in $\mathrm{x}$ - and $\mathrm{y}$-axis. The red numbers indicate the points inside each quadrant (Day 1, 2 and 3 interval are explained in Table 2 caption).
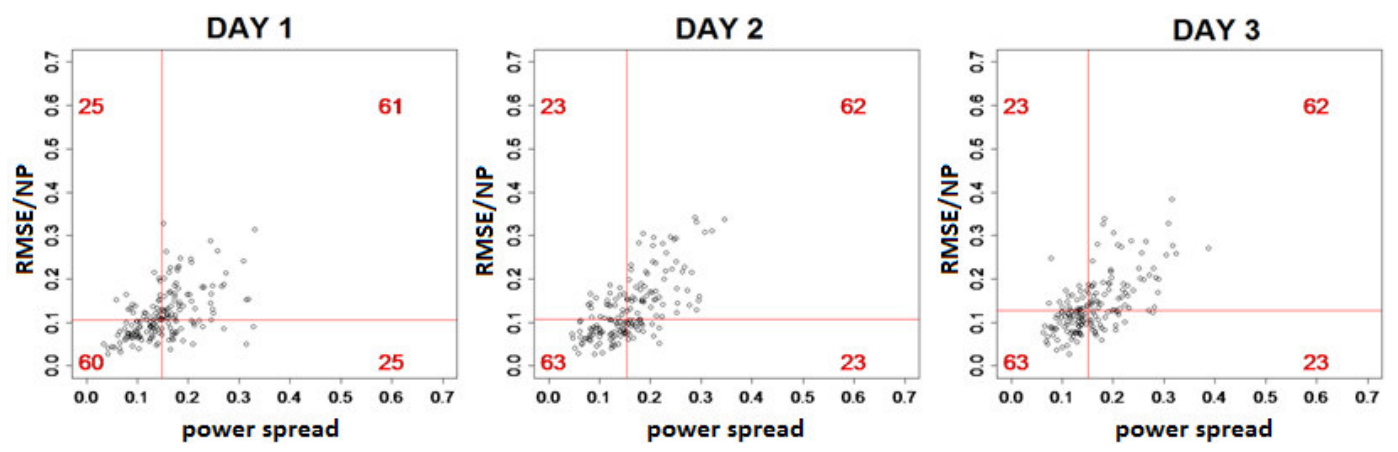

Figure 3. ECMWF deterministic power forecast error (RMSE/NP) vs. ensemble power spread computed as a daily average for three lead times. EPS T639/T319 $(32 \mathrm{~km})$. The lines are plotted on the statistical median. The red numbers indicate the points inside each quadrant (Day 1, 2 and 3 interval are explained in Table 2 caption).

indices (Pearson) and ratio between cases falling simultaneously above or below their respective median value and total number of cases. Note that contingency tables only comprise one approach to assess the potentially existing spread-skill relationship for ensemble forecasts and that other approaches may be used in order to compare several ensemble forecasting systems for the wind power application. As an example, Pinson et al. (2009) compared ensemble forecasts of wind power based on lagged-averaged poor man's ensembles, as well as NCEP and ECMWF ensemble predictions. The results presented here are not directly comparable, even though our analysis and conclusions are qualitatively similar. More work is required in the future for the thorough comparison of rival approaches to ensemble forecasting of wind power generation based on different meteorological ensemble forecasts used as input.

\section{Deterministic approach using the ensemble mean}

We conducted a WPF in a deterministic approach using the calibrated ensemble mean and comparing the obtainable performances with those of the ECMWF deterministic model; Table 2 shows that for the low resolution case the re- sults are slightly worse, showing an increase in RMSE/NP and MAE/NP between 0.5 and $1 \%$. For 2011, however, the increase in resolution allows to obtain comparable performances between the power forecast based on high resolution deterministic meteorological model and on the ensemble mean.

\section{Conclusions}

In this work we showed that the increase in resolution of the ECMWF deterministic model does not necessary assure better performances of corresponding power forecasts in this test case. However, higher resolution of the EPS allowed us to obtain better performances in a WPF deterministic approach (using the ensemble mean as a deterministic model) than with lower resolution. Furthermore, the new ensemble spread seems to be usable as an indicator of a WPF accuracy with slightly better results than with the lower resolution, at least for the time horizon until $72 \mathrm{~h}$ ahead.

Acknowledgements. This work has been financed by the Research Fund for the Italian Electrical System under the Contract Agreement between RSE (formerly known as ERSE) and the 
Ministry of Economic Development - General Directorate for Nuclear Energy, Renewable Energy and Energy Efficiency stipulated on 29 July 2009 in compliance with the Decree of 19 March 2009.

The authors also acknowledge Federico Fioretti and Umberto Deangelis of Enel Spa for providing meteorological and power data for the test case.

Edited by: L. Wald

Reviewed by: three anonymous referees

The publication of this article is sponsored by the European Meteorological Society.

\section{References}

Alessandrini, S., Decimi, G., and Ronzio, D.: Studio della relazione energia producibile/prodotta per un campo eolico su terreno complesso con modelli meteorologici dinamici, Rapporto di Ricerca RSE no. 10000262, 2010.

Alessandrini, S., Pinson, P., Hagedorn, R., Decimi, G., and Sperati, S.: An application of ensemble/multi model approach for wind power production forecasting, Adv. Sci. Res., 6, 35-37, doi:10.5194/asr-6-35-2011, 2011.

Cali, Ue., Lange, B., Dobschinski, J., Kurt, M., Moehrlen, C., and Ernst, B.: Artificial neural network based wind power forecasting using a multi-model approach, 7th International Workshop on Large Scale Integration of Wind Power and on Transmission Networks for Offshore Wind Farms, Madrid, 2008.
ECMWF internal report: Horizontal resolution increase (www. ecmwf.int), 2010.

Giebel, G., Brownsword, R., Kariniotakis, G., Denhard, M., and Draxl, C.: The State of the Art in Short-Term Prediction of Wind Power, Anemos.plus deliverable report, 2011.

Holmgren, E., Siebert, N., and Kariniotakis, G.: Wind Power Prediction Risk Indices Based on Numerical Weather Prediction Ensembles, Proceedings of the EWEC 2010 Conference, Warsaw, Poland, 20-23 April, 2010.

Pinson, P.: Adaptive recalibration of ensemble forecasts of $(u, v)$ winds, Q. J. Roy. Meteorol. Soc., doi:10.1002/qj.1873, in press, 2012.

Pinson, P., Nielsen, H. A., Madsen, H., and Kariniotakis, G.: Skill forecasting from different wind power ensemble prediction methods, J. Phys. Conf. Ser., 75, doi:10.1088/17426596/75/1/012046, 2007.

Pinson, P., Nielsen, H. A., Madsen, H., and Kariniotakis, G.: Skill forecasting from ensemble predictions of wind power, Appl. Energ., 86, 1326-1334, 2009.

Von Bremen, L.: Combination of deterministic and probabilistic meteorological models to enhance wind farm forecast, J. Phys. Conf. Ser., 75, 012050, doi:10.1088/1742-6596/75/1/012050, 2007. 